\title{
Positron-induced Radiophotoluminescence in Ag-doped Glasses
}

\author{
Hirokazu Masai, ${ }^{1 *}$ Yuka Yanagida, ${ }^{2}$ Hiroki Kawamoto, ${ }^{3}$ \\ Yasuhiro Koguchi, ${ }^{2}$ Masanori Koshimizu, ${ }^{3}$ and Masato Yamawaki ${ }^{4}$ \\ ${ }^{1}$ National Institute of Advanced Industrial Science and Technology, \\ 1-8-31 Midorigaoka, Ikeda, Osaka 563-8577, Japan \\ ${ }^{2}$ Oarai Research Center, Chiyoda Technol Corporation, Oarai, Ibaraki 311-1313, Japan \\ ${ }^{3}$ Department of Applied Chemistry, Tohoku University, 6-6-07 Aramaki, Aoba, Sendai, Miyagi 980-8579, Japan \\ ${ }^{4}$ National Institute of Advanced Industrial Science and Technology, \\ 1-1-1 Umezono, Tsukuba, Ibaraki 305-8568, Japan
}

(Received October 13, 2021; accepted November 30, 2021)

Keywords: positron, glass, silver, radiophotoluminescence, electron spin resonance

Positron annihilation spectroscopy (PAS) is used for the quantification of cavities in a matrix. Although PAS is sometimes considered a nondestructive measurement method, it is worth investigating the interaction of positrons with the matrix during PAS. Here, we have demonstrated that defects are generated in silver-doped phosphate glasses during positron annihilation measurement and that radiophotoluminescence (RPL) is observed after irradiation. There is a linear correlation between the irradiation duration and the observed RPL intensity of the glasses. We observed RPL after a high irradiation dose even after conventional thermal annealing at $360{ }^{\circ} \mathrm{C}$ for $1 \mathrm{~h}$. The formation of $\mathrm{Ag}^{2+}$ species detectable by electron spin resonance (ESR) was confirmed. From the concentration of ESR-active $\mathrm{Ag}^{2+}$ and $\mathrm{Ag}_{2}{ }^{+}$species, it is expected that approximately $0.1 \%$ of $\mathrm{Ag}^{+}$cations were changed after 5 days of positron irradiation.

\section{Introduction}

Recently, various spectroscopic techniques have been widely used to investigate the structural and physical properties of materials. One of the spectroscopic techniques is positron annihilation spectroscopy (PAS), which can quantitatively evaluate cavities with sizes from $10 \mathrm{~nm}$ to sub-nanometer by detecting the $\gamma$-rays $(511 \mathrm{keV})$ emitted when positrons are annihilated. The existence of positrons was first reported by Anderson and Neddermeyer in $1933,{ }^{(1)}$ and the use of positron emission tomography as a diagnostic method for cancer is a typical medical application of PAS. ${ }^{(2-4)}$ PAS can be used to evaluate both open and closed cavities in various types of material. ${ }^{(5-10)}$ Although positron annihilation measurement has conventionally been performed in quantum beam facilities or radiation-controlled areas, a commercial instrument that can be used in the laboratory has been developed to make general measurements possible. ${ }^{(11)}$

*Corresponding author: e-mail: hirokazu.masai@aist.go.jp

https://doi.org/10.18494/SAM3688 
PAS is sometimes considered a nondestructive measurement method. However, we have found that color centers in FD-7 phosphate glass are generated after PAS analysis. This glass has been used as glass badges for personal monitoring provided by Chiyoda Technol Corporation. ${ }^{(12-21)}$ It has been reported that the glass system exhibits radiophotoluminescence (RPL), ${ }^{(21-26)}$ a luminescence owing to electronic transitions within luminescent centers that are generated in phosphors by ionizing radiation. Radiation-induced effects in solid materials have been reported in a variety of fields. In particular, it is often observed that defects are more easily formed by the interaction of radiation and matter in glasses than in crystals. ${ }^{(27-31)}$ On the other hand, it has also been reported that the generated defects are eliminated by thermal annealing. ${ }^{(14)}$ Therefore, not only the effect of positron irradiation but also its reversibility by thermal annealing is examined in the present study. We examine the relationship between positron radiation and the RPL behavior of FD-7 glass. In addition, defect formation and reversibility during thermal annealing, which are characteristic behaviors of Ag-doped phosphate glasses, are discussed.

\section{Materials and Methods}

FD-7 glass samples, whose size was $33 \times 7 \times 1 \mathrm{~mm}^{3}$, were supplied by Chiyoda Technol Corporation. The chemical composition of the FD-7 glass was $0.09 \mathrm{Ag}_{2} \mathrm{O}-27.73 \mathrm{Na}_{2} \mathrm{O}-$ $13.14 \mathrm{Al}_{2} \mathrm{O}_{3}-59.04 \mathrm{P}_{2} \mathrm{O}_{5}$ (mol\%). ${ }^{(17)}$ Positron irradiation was performed using a PSA Type L-II system (Toyo Seiko Co., Ltd.) with an anti-coincident system. ${ }^{(11)}$ The glass samples were directly placed on ${ }^{22} \mathrm{Na}$ isotope sealed in Kapton $(<1 \mathrm{MBq})$ with a diameter of $15 \mathrm{~mm}$ at room temperature (RT) without a spacer. During the measurement, dry $\mathrm{N}_{2}$ gas was flowed at a flow rate of $80 \mathrm{cc} / \mathrm{min}$. Two or three spots were irradiated for each duration of positron irradiation. Optical absorption spectra were measured using an UH-4150 spectrophotometer (Hitachi HighTech.). The refractive index at $633 \mathrm{~nm}$ was measured with a prism coupler (Metricon). PL spectra were recorded at RT using an F7000 fluorescence spectrophotometer (Hitachi High-Tech.) with a G475 glass filter. RPL intensity was monitored using an FGD-670 RPL dosimeter reader (AGC Techno Glass Co.). Electron spin resonance (ESR) measurement was performed with a JES-X330 (JEOL) spectrometer at a microwave frequency, field, and power of $9444 \mathrm{MHz}, 0.8 \mathrm{mT}$, and 6 $\mathrm{mW}$, respectively. The area irradiated by microwaves during ESR measurement was from the bottom of the sample tube to a height of $43.5 \mathrm{~mm}$. To calculate the concentration of ESR-active species, a measurement was also performed on 2,2,6,6-tetramethylpiperidine 1-oxyl (TEMPO) solution $\left(3.00 \times 10^{-7} \mathrm{~mol} / \mathrm{L}\right.$ of $160 \mu \mathrm{L} n$-hexane).

\section{Results and Discussion}

FD-7 glass is transparent, and no absorption band was observed in the visible region before irradiation. The refractive index of the FD-7 glass at $633 \mathrm{~nm}$ was 1.510(4). From the PAS, it was found that the $i_{3}$ and $\tau_{3}$ values of the FD-7 glass were $13.6 \%$ and $1.078 \mathrm{~ns}$, respectively. The cavity radius calculated from the $\tau_{3}$ value was $0.1784 \mathrm{~nm}$. After positron irradiation for 10 days, the irradiated part was colored, indicating that color centers were generated by the irradiation. 
The colored region can be quantified from the optical absorption spectrum. Figure 1(a) shows a comparison of the optical absorption spectra of samples irradiated with positrons for 5 and 10 days and a non-irradiated sample. Figure 1(b) shows differential optical absorption spectra of the two irradiated FD-7 glass samples, which were obtained by subtracting the spectrum of the nonirradiated FD-7 glass. There is a broad absorption band located at $3.9 \mathrm{eV}$, which is attributed to defects in the glass, which was also suggested by X-ray-induced defects in this glass in a previous study. ${ }^{(14)}$

Many researchers have reported the RPL behavior of FD-7 glass after radiation. ${ }^{(12-21)}$ In the RPL mechanism, the generated Ag species are the origin of the luminescence. Figure 2 shows PL spectra of the front (solid line) and back (dotted line) of FD-7 glass after positron irradiation for 5 days. The excitation energy was $3.91 \mathrm{eV}(317 \mathrm{~nm})$, which corresponds to the generated absorption band shown in Fig. 1. The broad emission band is similar to that reported in previous papers. The intensity of the PL spectrum of the front is higher than that of the back. Therefore, it is expected that the concentration of defects at the surface is higher than that inside the glass. The $\gamma$-ray energy distribution of ${ }^{22} \mathrm{Na}$ should show two peaks, a peak of $1274.5 \mathrm{keV}$ and a peak of $511 \mathrm{keV}$, due to positron annihilation. Although we cannot precisely separate the contribution of $\gamma$-rays from those of ${ }^{22} \mathrm{Na}$ and positron annihilation, the main origin of the defect formation is the $\beta^{+}$reaction of positrons from the ${ }^{22} \mathrm{Na}$.

To examine the relationship between irradiation duration and emission intensity, we measured the RPL intensity of the FD-7 glass samples after different durations. Figure 3 shows the relationship between the irradiation duration of positrons and the RPL intensity of FD-7 glass samples, which was monitored using an RPL dosimeter reader. The durations were 1, 10, $100,1000,10000$, and $43200 \mathrm{~s}(12 \mathrm{~h})$ at $25^{\circ} \mathrm{C}$. No data for irradiation of more than $43200 \mathrm{~s}$ is shown in the figure because the count of the RPL intensity was over the maximum limit of the equipment (9999999). It has been reported that there is a linear relationship between the irradiation dose and the RPL intensity up to a dose of $3000 \mathrm{R}(\sim 26 \mathrm{~Gy}){ }^{\left({ }^{(21)}\right.}$ When a sample was irradiated with $6 \mathrm{mGy}$ of Cs from a distance of $2 \mathrm{~m}$ in air, the monitored RPL intensity was

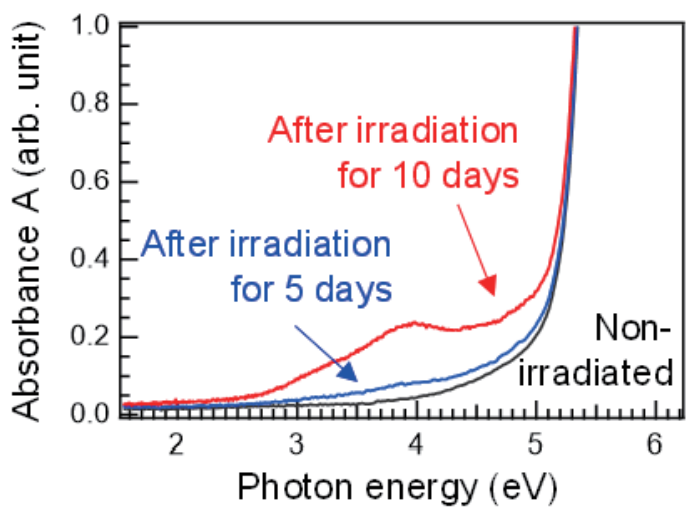

(a)

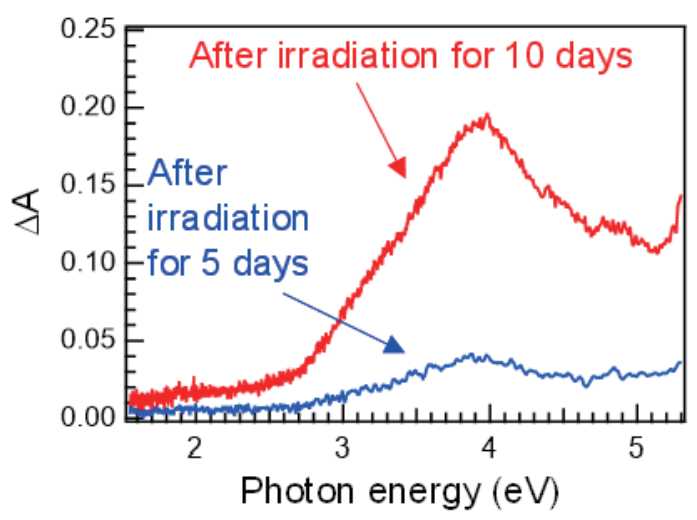

(b)

Fig. 1. (Color online) (a) Optical absorption spectra of FD-7 glass samples after positron irradiation from 22Na for 5 and 10 days along with that of non-irradiated FD-7 glass. (b) Changes in optical absorption spectra of FD-7 glass after positron irradiation for 5 and 10 days. 


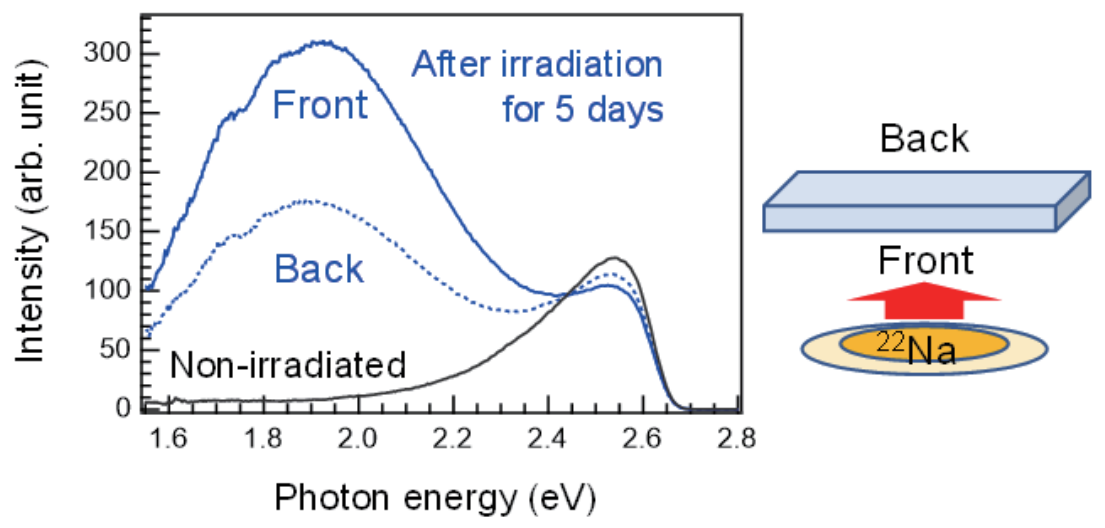

Fig. 2. (Color online) PL spectra of front (solid line) and back (dotted line) of FD-7 glass after positron irradiation for 5 days. The PL spectrum of non-irradiated FD-7 glass is also shown for comparison. The excitation energy was $3.91 \mathrm{eV}(317 \mathrm{~nm})$.

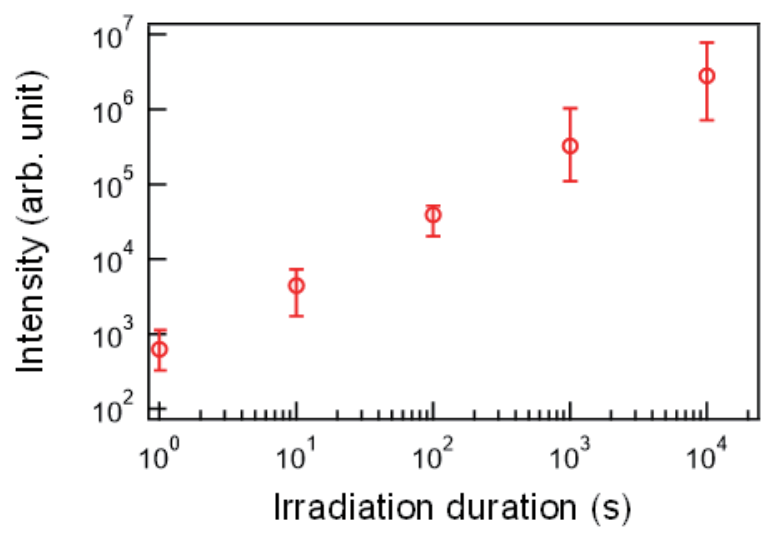

Fig. 3. (Color online) Luminescence intensity of FD-7 glass samples as a function of irradiation duration.

approximately 5400 counts. Assuming that a linear relationship between dose and RPL count can be applied to the data in Fig. 3, the dose in the overflow region (9999999) was estimated as $11 \mathrm{~Gy}$. Note that this overflow was due to the limitation of the measurement device, rather than originating in the glass badge itself. With increasing irradiation duration, the emission intensity monotonically increased. Also note that the RPL intensity of the non-irradiated sample was less than $10^{2}$, which clearly shows the effect of even $1 \mathrm{~s}$ of irradiation.

It has been reported that the irradiation-induced changes in Ag-doped phosphate glasses can be reversed by thermal annealing. To confirm the thermal reversibility, the RPL intensity of the glass samples after annealing was also measured. Figure 4 shows the RPL intensities of the FD-7 glass samples before irradiation, after the irradiation of positrons, and after conventional annealing at $360{ }^{\circ} \mathrm{C}$ for $1 \mathrm{~h}$. The inset shows a schematic image of the irradiated part and the non-irradiated part. The RPL intensity of the non-irradiated part after irradiation refers to the RPL intensity from the region of the FD-7 glass plate that was not directly irradiated. After annealing, the intensity of the control sample returned to the original value. In contrast, the 


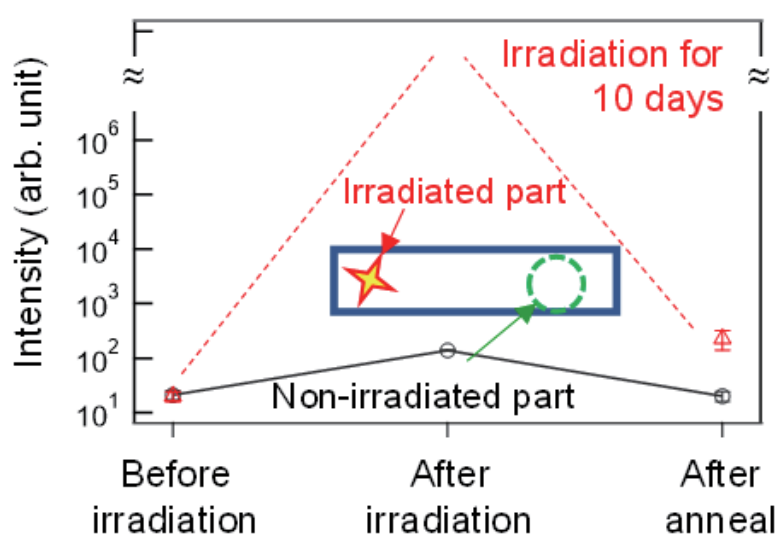

Fig. 4. (Color online) Luminescence intensity of FD-7 glass samples before and after positron irradiation for 10 days and after annealing at $360^{\circ} \mathrm{C}$ for $1 \mathrm{~h}$ in an ambient atmosphere. The inset shows a schematic image of the irradiated part and the non-irradiated part in a glass.

intensity of the FD-7 glass after 10 days of positron irradiation did not return to the original value, suggesting that part of the defects were not eliminated by the thermal annealing at $360{ }^{\circ} \mathrm{C}$ for $1 \mathrm{~h}$.

The presence of luminescent centers after thermal annealing is thought to be due to the high irradiation dose. Therefore, we examined the effect of thermal annealing on the RPL intensity after several annealing processes. Figure 5 shows the RPL intensity of several FD-7 glasses as a function of annealing time, where the irradiation periods were 3, 5, and 10 days. For the FD-7 glasses irradiated for 3 and 5 days, the RPL intensity decreased to the same level as that of the non-irradiated glass after three annealing cycles. On the other hand, another annealing process was needed for the sample irradiated for 10 days to decrease the RPL intensity to less than $10^{2}$.

Finally, we examined the ESR of the FD-7 glass subjected to positron irradiation for 5 days to discuss the defect formation by the irradiation. Figure 6 shows the ESR spectrum of the FD-7 glass after 5 days of irradiation. The signals at 304-317, 319-335, and 362-374 mT correspond to peaks with $g=2.18,2.07$, and 1.83 , respectively. The ESR signal in the positron-irradiated glass confirms the generation of non- $\mathrm{Ag}^{+}$species, which was previously suggested by the results of optical absorption and RPL measurement. In the previous papers, ${ }^{(13,15,31)}$ the signal at $g=2.07$ was assigned to $\mathrm{Ag}^{2+}$, whereas the signals at $g=2.18$ and 1.83 correspond to $\mathrm{Ag}_{2}{ }^{+}$(a cluster of $\mathrm{Ag}^{+}$and $\left.\mathrm{Ag}^{0}\right)$. From the weight $(42.4 \mathrm{mg})$ and density $\left(2.6 \mathrm{~g} / \mathrm{cm}^{3}\right)$ of the sample used for ESR measurement, its volume was calculated to be $16.3 \mathrm{~mm}^{3}$. The amount of each Ag species can be calculated as double the integral value for TEMPO, and the amounts of $\mathrm{Ag}^{2+}$ and $\mathrm{Ag}_{2}{ }^{+}$were calculated to be $1.8 \times 10^{-10}$ and $3.3 \times 10^{-10} \mathrm{~mol}$, respectively. Therefore, the concentrations of $\mathrm{Ag}^{2+}$ and $\mathrm{Ag}_{2}{ }^{+}$were calculated to be $1.1 \times 10^{-11}$ and $2.0 \times 10^{-11} \mathrm{~mol} / \mathrm{mm}^{3}$, respectively. In a conventional RPL mechanism, both $\mathrm{Ag}^{2+}$ and $\mathrm{Ag}^{0}$ are generated by irradiation. Therefore, the similar concentrations of these $\mathrm{Ag}$ species are consistent with the conventionally proposed mechanism. On the other hand, from the molecular weight of the FD-7 glass $(114.5 \mathrm{~g} / \mathrm{mol})$, the total concentration of Ag cations was also calculated to be $2.0 \times 10^{-8} \mathrm{~mol} / \mathrm{mm}^{3}$. On the basis of these concentrations, it is expected that approximately $0.1 \%$ of the $\mathrm{Ag}^{+}$cations were changed 


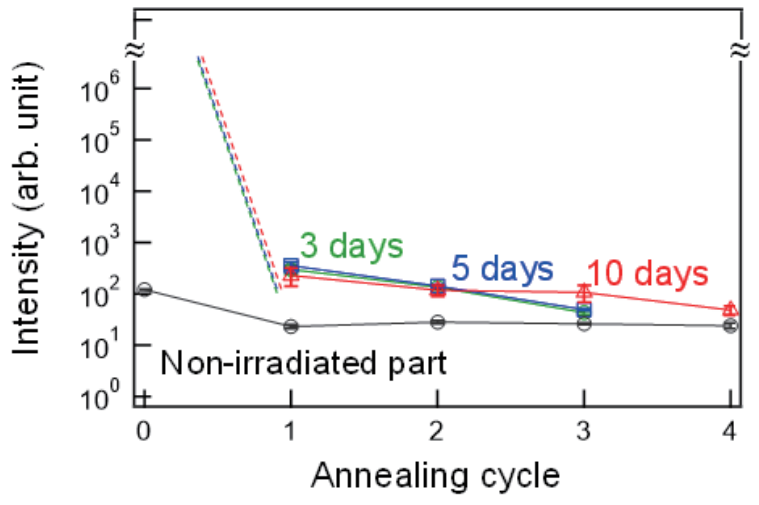

Fig. 5. (Color online) RPL intensity of FD-7 glass samples as a function of annealing cycle. The annealing condition was $360{ }^{\circ} \mathrm{C}$ for $1 \mathrm{~h}$ in an ambient atmosphere.

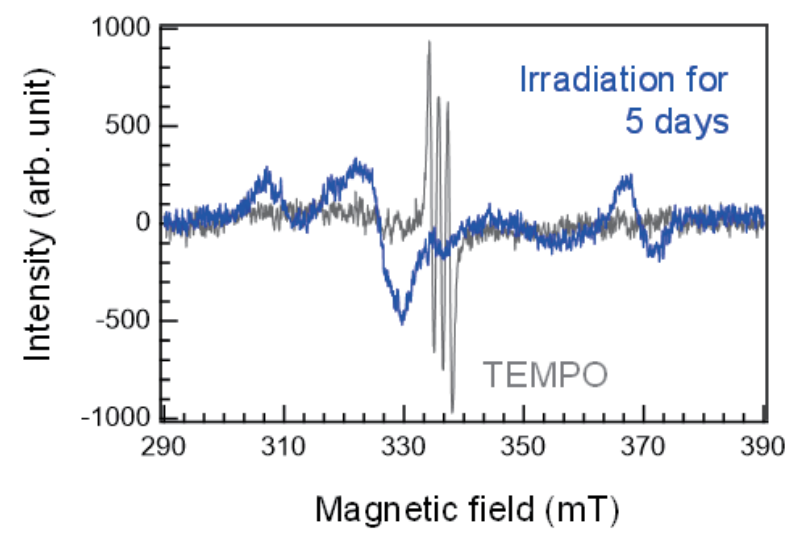

Fig. 6. (Color online) ESR spectrum of FD-7 glass after 5 days of positron irradiation. The spectrum of TEMPO, which was used to calculate the concentration, is also shown.

after the 5 days of irradiation. This small change in the number of Ag species is consistent with a previous observation that only the generated peak of a $\mathrm{Ag}-\mathrm{L}_{3}$ edge X-ray absorption near-edge structure spectrum was changed by X-ray irradiation and that the remainder of the spectrum remained unchanged. ${ }^{(17)}$

\section{Conclusions}

We have found that positron irradiation induces defect formation in Ag-doped phosphate glasses. The observation of RPL suggests that irradiated positrons interact with the glass matrix even in a small radiation time. Although most of the generated defects were eliminated by a conventional annealing process, residual defects were also observed after annealing, depending on the irradiation dose. The existence of $\mathrm{Ag}^{2+}$ and $\mathrm{Ag}^{0}$ defects, whose concentrations were on the order of $0.1 \%$ of that of the $\mathrm{Ag}^{+}$cations, was confirmed.

\section{References}

1 C. D. Anderson and S. H. Neddermeyer: Phys. Rev. 43 (1933) 1034.

2 Y. L. Yamamoto, C. Thompson, and E. Meyer: J. Nucl. Med. 17 (1976) 546.

3 M. E. Raichle, A. M. MacLeod, A. Z. Snyder, W. J. Powers, D. A. Gusnard, and G. L. Shulman: Proc. Natl. Acad. Sci. USA 98 (2001) 676.

4 A. G. Devolder, A. M. Goffinet, A. Bol, C. Michel, T. Debarsy, and C. Laterre: Arch. Neurol. 47 (1990) 197.

5 N. West: Adv. Phys. 22 (1973) 263.

6 R. A. Pethrick: Prog. Polym. Sci. 22 (1997) 1.

7 M. Ono, K. Hara, M. Fujinami, and S. Ito: Appl. Phys. Lett. 101 (2012) 164103.

8 H. Masai, Y. Onodera, S. Kohara, T. Ohkubo, A. Koreeda, Y. Fujii, M. Koshimizu, and M. Yamawaki: Phys. Status Solidi B 257 (2020) 2000186.

9 H. Masai, T. Ohkubo, Y. Fujii, A. Koreeda, T. Yanagida, T. Ina, and K. Kintaka: Sci. Rep. 11 (2021) 3811.

10 H. Masai, H. Kimura, M. Akatsuka, T. Kato, N. Kitamura, and T. Yanagida: J. Lumin. 241 (2022) 118481.

11 M. Yamawaki, Y. Kobayashi, K. Hattori, and Y. Watanabe: Jpn. J. Appl. Phys. 50 (2011) 086301.

12 R. Yokota and H. Imagawa: J. Phys. Soc. Jpn. 20 (1965) 1537. 
13 R. Yokota and H. Imagawa: J. Phys. Soc. Jpn. 23 (1966) 1038.

14 Y. Miyamoto, Y. Takei, H. Nanto, T. Kurobori, A. Konnai, T. Yanagida, A. Yoshikawa, Y. Shimotsuma, M. Sakakura, K. Miura, K. Hirao, Y. Nagashima, and T. Yamamoto: Rad. Meas. 46 (2011) 1480.

15 S. W. S. McKeever, S. Sholom, and N. Shrestha: Radiat. Meas. 123 (2019) 13.

16 H. Kawamoto, Y. Fujimoto, M. Koshimizu, G. Okada, T. Yanagida, and K. Asai: Jpn. J. Appl. Phys. 57 (2018) 062401.

17 H. Masai, M. Koshimizu, H. Kawamoto, T. Ohkubo, A. Koreeda, Y. Fujii, K. Ohara, H. Ofuchi, and H. Setoyama: J. Ceram. Soc. Jpn. 127 (2019) 924.

18 H. Kawamoto, H. Tanaka, M. Koshimizu, Y. Fujimoto, and K. Asai: Nucl. Inst. Method Phys. Res., Sec. B 479 (2020) 137.

19 D. Y. C. Huang and S.-M. Hsu: Radiophoto-luminescent glass dosimeter: Advances in Cancer Therapy (IntechOpen, 2011) p. 553.

20 D. Maki, M. Ube, Y. Nagashima, W. Shinozaki, S. Ueno, Y. Koguchi, and N. Takeuchi: Radiat. Prot. Dosim. 171 (2016) 337.

21 T. Omori, M. Osada, T. Ikegami, and N. Nishida: Toshiba Rev. 37 (1982) 79 (in Japanese).

22 J. H. Schulman, R. J. Ginter, and C. C. Klick: J. Appl. Phys. 22 (1951) 1479.

23 J. Qiu and K. Hirao: J. Mater. Sci. Lett. 20 (2001) 691.

24 L. Skuja: J. Non-Cryst. Solids 239 (1998) 16.

25 T. Kato, D. Shiratori, M. Iwao, H. Takase, D. Nakauchi, N. Kawaguchi, and T. Yanagida: Sens. Mater. 33 (2021) 2163.

26 H. Tanaka, Y. Fujimoto, M. Koshimizu, T. Yanagida, T. Yahaba, K. Saeki, and K. Asai: Sens. Mater. 28 (2016) 863.

27 D. L. Griscom: J. Non-Cryst. Solids 357 (2011) 1945.

28 D. L. Griscom, P. C. Taylor, D. A. Ware, and P. J. Bray: J. Chem. Phys. 48 (1968) 5158.

29 G. Pfeiffer, M. A. Paesler, and S. C. Agarwal: J. Non-Cryst. Solids 130 (1991) 111.

30 H. Masai, G. Okada, N. Kawaguchi, and T. Yanagida: Opt. Mater. Express 9 (2019) 2037.

31 N. I. Mel'nikov, D. P. Peregood, and R. A. Zhitnikov: J. Non-Cryst. Solids 16 (1974) 195. 